Canad. Math. Bull. Vol. 56 (1), 2013 pp. 213-217

http://dx.doi.org/10.4153/CMB-2011-146-4

(C) Canadian Mathematical Society 2011

\title{
A Locally Compact Non Divisible Abelian Group Whose Character Group Is Torsion Free and Divisible
}

\author{
Daniel V. Tausk
}

Abstract. It was claimed by Halmos in 1944 that if $G$ is a Hausdorff locally compact topological abelian group and if the character group of $G$ is torsion free, then $G$ is divisible. We prove that such a claim is false by presenting a family of counterexamples. While other counterexamples are known, we also present a family of stronger counterexamples, showing that even if one assumes that the character group of $G$ is both torsion free and divisible, it does not follow that $G$ is divisible.

\section{Introduction}

Let $G$ be an abelian group! Given an integer $n$, we consider the subgroups of $G$ defined by

$$
n G=\{n x: x \in G\}, \quad G[n]=\{x \in G: n x=0\} .
$$

If $G$ is an abelian topological group, then its character group $\widehat{G}$ is the abelian group of all continuous homomorphisms $\xi: G \rightarrow S^{1}$, where $S^{1}$ is the (multiplicative) circle group of unitary complex numbers; the group $\widehat{G}$ is endowed with the compact-open topology. The celebrated Pontryagin duality theorem (see, for instance, [7]) states that if $G$ is a Hausdorff locally compact abelian topological group, then its character group $\widehat{G}$ is a Hausdorff locally compact abelian topological group as well and the character group of $\widehat{G}$ is $G$ itself; more precisely, the map that associates each $x \in G$ with the evaluation map $\widehat{G} \ni \xi \mapsto \xi(x) \in S^{1}$ is a homeomorphic isomorphism between $G$ and the character group of $\widehat{G}$.

If $H$ is a subgroup of $G$, then the annihilator of $H$ is the subgroup $\operatorname{ann}(H)$ of $\widehat{G}$ consisting of all characters $\xi: G \rightarrow S^{1}$ that are trivial over $H$. Clearly, given an integer $n$,

$$
\operatorname{ann}(n G)=\widehat{G}[n] .
$$

In particular, if $G$ is divisible, i.e., if $n G=G$ for every nonzero integer $n$, then its character group $\widehat{G}$ is torsion free, i.e., $\widehat{G}[n]$ is trivial for every nonzero integer $n$. It was claimed by Halmos [5] that the converse is true if $G$ is Hausdorff locally compact. The argument presented in [5] has a gap: if $\widehat{G}$ is torsion free, then $\operatorname{ann}(n G)$ is trivial for every nonzero integer $n$, but that, in principle, implies only that $n G$ is dense 2 in

Received by the editors March 3, 2010; revised January 9, 2011.

Published electronically July 9, 2011.

AMS subject classification: 22B05.

${ }^{1}$ Except for the circle group $S^{1}$, abelian groups will be written additively.

2 If $\operatorname{ann}(n G)$ is trivial, then $n G$ is indeed dense in $G$. Otherwise, Pontryagin duality would give us a nontrivial character on the (nontrivial) quotient of $G$ by the closure of $n G$ and such nontrivial character would correspond to a nontrivial element of $\operatorname{ann}(n G)$. 
$G$, not that $n G=G$. It should be observed, however, that the claim made by Halmos is true if $G$ is either compact or discrete, and that the proof of his main result is not affected by the incorrect claim.

In Section 3, we will present a family of examples of Hausdorff locally compact abelian topological groups $G$ such that $n G$ is dense in $G$ for every nonzero integer $n$, but such that $n G \neq G$ for some nonzero integer $n$. In particular, any such group $G$ is an example of a Hausdorff locally compact abelian topological group that is not divisible, but whose character group is torsion free. While other examples of that phenomenon are known (see [1, 4.16]), in Section 4 we will also present a family of examples of Hausdorff locally compact abelian topological groups $G$ that are both divisible and torsion free, but such that $\widehat{G}$ is (torsion free but) not divisible. In particular, by Pontryagin duality, it follows that $\widehat{G}$ is a Hausdorff locally compact abelian topological group whose character group (which is isomorphic to $G$ ) is both divisible and torsion free, but still $\widehat{G}$ is not divisible.

\section{Extending the Topology of a Subgroup}

Let us start by presenting a general construction of a topology on an abelian group from a topology on a given subgroup (the construction is well known; see, for instance, [2-4]). Let $G$ be an abelian group and $H$ be a subgroup of $G$. Assume that $H$ is endowed with a topology that makes it into a topological group. We claim that there exists a unique topology on $G$ such that

(i) $G$ is a topological group;

(ii) the given topology of $H$ is inherited from $G$;

(iii) $H$ is open in $G$.

Such a topology is constructed as follows. Given $g \in G$, the coset $g+H$ of $H$ can be endowed with a topology by requiring that the translation map

$$
L_{g}: H \ni x \longmapsto g+x \in g+H
$$

be a homeomorphism. The fact that the translation maps of $H$ are homeomorphisms of $H$ implies that the topology defined on the coset $g+H$ does not depend on the representative $g$ of the coset. We topologize $G$ by making it the topological sum of the cosets $g+H, g \in G$. That is, we say that $U$ is open in $G$ if $U \cap(g+H)$ is open in $g+H$ for every $g \in G$. One readily checks that such a topology is the only topology on $G$ satisfying (i), (ii), and (iii). Notice that since the cosets of $H$ are all homeomorphic to $H$ and open in $G$, it follows that if $H$ is Hausdorff, then so is $G$. Moreover, since every compact neighborhood of the neutral element in $H$ is also a compact neighborhood of the neutral element in $G$, it follows that $G$ is locally compact if $H$ is locally compact.

\section{The First Family of Counterexamples}

Let $A$ be a Hausdorff compact abelian topological group that is not divisible, and let $B$ be a divisible abelian group such that $A$ is a subgroup of $B$ (for instance, let $B=S^{1}$ and $A$ be a nontrivial finite subgroup of $S^{1}$ endowed with the discrete topology). 
Let $B^{\omega}$ denote the group of all sequences $\left(x_{k}\right)_{k \in \omega}$ of elements of $B$ and let $G$ denote the subgroup of $B^{\omega}$ consisting of those sequences $\left(x_{k}\right)_{k \in \omega}$ such that $x_{k}$ is in $A$ for $k$ sufficiently large. Let $H=A^{\omega}$ denote the subgroup of $G$ consisting of sequences in $A$. We endow $H$ with the product topology and $G$ with the unique topology satisfying (i), (ii), and (iii) of Section 2 , Then $H$ is a Hausdorff compact topological group and thus $G$ is a Hausdorff locally compact topological group. If $n$ is a nonzero integer, then the subgroup $n G$ of $G$ consists of those sequences $\left(x_{k}\right)_{k \in \omega}$ such that $x_{k}$ is in $n A$ for $k$ sufficiently large. If $n_{0}$ is a nonzero integer such that $n_{0} A \neq A$, then $n_{0} G \neq G$ and therefore $G$ is not divisible. We will show that if $n$ is a nonzero integer, then $n G$ is dense in $G$ and from this it will follow from the discussion in the introduction that the character group $\widehat{G}$ is torsion free. Let $J$ denote the subgroup of $G$ consisting of sequences $\left(x_{k}\right)_{k \in \omega}$ in $B$ that are trivial for $k$ sufficiently large. Since $J$ is obviously contained in $n G$ for any nonzero integer $n$, it suffices to prove that $J$ is dense in $G$ in order to establish that $n G$ is dense in $G$ for every nonzero integer $n$. Clearly, $G=H+J$, so that $J$ intersects every coset of $H$. Now let us prove that $J$ is dense in $G$ by proving that $J \cap(x+H)$ is dense in $x+H$ for every coset $x+H$ of $H$ in $G$. Since the coset $x+H$ intersects $J$, we can assume that $x \in J$. Thus, the translation map $L_{x}: H \rightarrow x+H$ is a homeomorphism that carries $J \cap H$ to $J \cap(x+H)$. From the definition of the product topology, it is obvious that $J \cap H$ is dense in $H$ and therefore $J \cap(x+H)$ is dense in $x+H$. This concludes the proof that the subgroup $J$ is dense in $G$.

\section{The Family of Stronger Counterexamples}

We will now present an example of a Hausdorff locally compact abelian topological group $G$ that is both divisible and torsion free, but such that its character group $\widehat{G}$ is not divisible. We need some preliminary lemmas.

Lemma 1 Let $G$ be an abelian divisible topological group. If there exists an open subgroup $H$ of $G$, a nonzero integer $n$, and a discontinuous homomorphism $\phi: H \rightarrow S^{1}$ that is trivial over $n H$, then the character group $\widehat{G}$ is not divisible.

Proof Since $S^{1}$ is divisible, $\phi$ extends to a (obviously discontinuous) homomorphism $\phi^{\prime}: G \rightarrow S^{1}$. Consider the homomorphism $\xi: G \rightarrow S^{1}$ defined by $\xi(x)=$ $\phi^{\prime}(n x)$, for all $x \in G$. Then $\xi$ is trivial over $H$ and, since $H$ is open, $\xi$ is continuous. Assuming by contradiction that $\widehat{G}$ is divisible, we can find a continuous homomorphism $\alpha: G \rightarrow S^{1}$ such that $\alpha(x)^{n}=\alpha(n x)=\xi(x)$ for all $x \in G$. Then $\alpha$ and $\phi^{\prime}$ are equal over $n G$ and since $G$ is divisible, we obtain that $\alpha=\phi^{\prime}$, contradicting the continuity of $\alpha$.

Lemma 2 Let $K$ be an abelian group endowed with a topology. If $K$ admits a proper dense subgroup $D$, then there exists a discontinuous homomorphism from $K$ to $S^{1}$.

Proof Since $K / D$ is a nontrivial abelian group, there exists a nontrivial homomorphism $\phi: K / D \rightarrow S^{1}$ (start with a nontrivial $S^{1}$-valued homomorphism defined over

\footnotetext{
${ }^{3}$ It is not necessary that $K$ be a topological group, i.e., the continuity of the operations of $K$ is not used in the proof.
} 
a nontrivial cyclic subgroup of $K / D$ and then extend it to all of $K / D$ using the fact that $S^{1}$ is divisible). The composition of $\phi$ with the quotient map $K \rightarrow K / D$ is a nontrivial homomorphism that is trivial over $D$, and therefore it must be discontinuous.

Corollary 3 Let $G$ be an abelian divisible topological group. If there exists an open subgroup $H$ of $G$ and a nonzero integer $n$ such that $H / n H$ (endowed with the quotient topology) has a proper dense subgroup, then the character group $\widehat{G}$ is not divisible.

Proof By Lemma 2, there exists a discontinuous $S^{1}$-valued homomorphism over $H / n H$; its composition with the quotient map $H \rightarrow H / n H$ is a discontinuous $S^{1}$-valued homomorphism over $H$ that is trivial over $n H$. The conclusion follows from Lemma1

The construction of our family of stronger counterexamples goes as follows. Let $A$ be a Hausdorff compact abelian non divisible topological group and let $B$ be a torsion free divisible abelian group such that $A$ is a subgroup of $B$. A concrete example of groups $A, B$ satisfying the required conditions will be supplied at the end of the section. Let $H=A^{\omega}$ denote the group of all sequences in $A$ endowed with the product topology, and let $G=B^{\omega}$ be the group of all sequences in $B$, endowed with the unique topology satisfying (i), (ii), and (iii) of Section 2 . The group $H$ is Hausdorff compact and thus $G$ is Hausdorff locally compact; moreover, like $B$, the group $G$ is both divisible and torsion free. We use Corollary 3 to establish that the character group $\widehat{G}$ is not divisible. Let $n$ be a nonzero integer such that $n A \neq A$. We claim that if $H / n H$ is endowed with the quotient topology, then it has a proper dense subgroup. First, we check that the quotient topology of $H / n H$ coincides with the product topology of $(A / n A)^{\omega}$, each factor $A / n A$ being endowed with the quotient topology. Namely, if $A / n A$ is endowed with the quotient topology, then the quotient map $A \rightarrow A / n A$ is continuous, open, and surjective; therefore, if $H / n H \cong(A / n A)^{\omega}$ is endowed with the product topology, then the quotient map $H \rightarrow H / n H$ is also continuous, open, and surjective and therefore it is a topological quotient map. This observation proves that the product topology of $(A / n A)^{\omega}$ coincides with the quotient topology of $H / n H$. Now it follows directly from the definition of the product topology that the subgroup of $H / n H \cong(A / n A)^{\omega}$ consisting of sequences $\left(x_{k}\right)_{k \in \omega}$ that are trivial for $k$ sufficiently large is a (proper) dense subgroup. This concludes the proof that $\widehat{G}$ is not divisible.

Finally, let us present a concrete example of groups $A, B$ satisfying the required conditions. Let $A$ be the group of $p$-adic integers (where $p$ is some fixed prime number) and $B$ be the $p$-adic field. We have $p A \neq A$, so that $A$ is not divisible; moreover, $B$ is a field of characteristic zero, so that it is both torsion free and divisible as an abelian group. The fact that $A$ can be made into a Hausdorff compact topological group follows from the observation that $A$ is (isomorphic to) the character group of the discrete $p$-quasicyclic group $\mathbb{Z}\left(p^{\infty}\right)$ of elements of $S^{1}$ whose order is a power of $p$ (see, for instance, [6, Proposition 3.1]) and that the character group of a discrete topological group is compact. 


\section{References}

[1] D. L. Armacost, The Structure of Locally Compact Abelian Groups. Monographs and Textbooks in Pure and Applied Mathematics 68. Marcel Dekker, 1981.

[2] B. Clark, M. Dooley, and V. Schneider, Extending topologies from subgroups to groups. Topology Proc. 10(1985), no. 2, 251-257.

[3] B. Clark and V. Schneider, The extending topologies. Internat. J. Math. Math. Sci. 7(1984), no. 3 , 621-623. http://dx.doi.org/10.1155/S0161171284000673

[4] The normal extensions of subgroup topologies Proc. Amer. Math. Soc. 97(1986), no. 1, 163-166. http://dx.doi.org/10.1090/S0002-9939-1986-0831407-9

[5] P. R. Halmos, Comment on the real line Bull. Amer. Math. Soc. 50(1944), 877-878. http://dx.doi.org/10.1090/S0002-9904-1944-08255-4

[6] D. K. Harrison, Infinite abelian groups and homological methods. Ann. of Math. 69(1959), 366-391. http://dx.doi.org/10.2307/1970188

[7] S. A. Morris, Pontryagin duality and the structure of locally compact abelian groups. London Mathematical Society Lecture Note Series 29. Cambridge University Press, Cambridge, 1977.

Departamento de Matemática, Universidade de São Paulo, Brazil

e-mail: tausk@ime.usp.br 